\title{
Avaliação e fatores associados à incapacidade funcional de idosos residentes em Instituições de longa permanência
}

\author{
Assessment and factors associated with functional disability of elderly \\ residents in long-term care facilities
}

\author{
Felipe Lima Rebêlo ${ }^{1}$ \\ D Carina Vasconcelos Gama Peixoto ${ }^{2}$ \\ Joaquim Silva Lima ${ }^{3}$ \\ Clara Maria de Araujo Silva ${ }^{4}$ \\ Alynne Iasmin Batista Santos ${ }^{5}$
}

Parecer de Aprovação do Comitê de Ética em Pesquisa do Centro Universitário (Cesmac), sob o número do parecer: 051406/2015.

Autor correspondente:

Felipe Lima Rebêlo

Rua Cônego Machado, 918, Farol

CEP: 57051-160, Maceió, Alagoas

+55 (82) 99974-9083

feliperebelo_fisio@yahoo.com.br

${ }^{1}$ Doutor em Ciências da Saúde pela Universidade Federal de Sergipe

Professor Titular II do curso de Fisioterapia do Centro Universitário Cesmac e Professor Assistente da Universidade Estadual de Ciências da Saúde de Alagoas

feliperebelo_fisio@yahoo.com.br

${ }^{2}$ Fisioterapeuta pelo Centro Universitário CESMAC

carinagama@hotmail.com

${ }^{3}$ Fisioterapeuta pelo Centro Universitário CESMAC

jokabelmiro@hotmail.com

${ }^{4}$ Graduanda em Fisioterapia na Universidade Estadual de Ciências da Saúde de Alagoas

cmaraujoft@gmail.com

${ }^{5}$ Graduanda em Fisioterapia na Universidade Estadual de Ciências da Saúde de Alagoas

iasminalynne@gmail.com

\section{Resumo}

Introdução: $O$ envelhecimento cursa com alterações funcionais que predispõe o idoso à perda funcional, que associada ao processo de institucionalização é potencializada.

Objetivo: Avaliar o nível de incapacidade funcional de idosos residentes em instituições de longa permanência em Maceió (AL).

Métodos: Estudo transversal realizado com os idosos residentes nas nove instituições da cidade de Maceió. Foram coletados dados socioeconômicos e demográficos e realizada a avaliação da capacidade funcional através do índice de Katz.

Resultados: A amostra final foi de 270 indivíduos. A média de idade foi de 78,9 anos, com predomínio do sexo feminino e baixo nível de escolaridade. A análise estatística evidenciou associação significativa entre uso de bengala/muleta ( $p<0,01$ ), síndrome do imobilismo ( $p<0,01$ ), menor frequência de visitas $(\mathrm{p}<0,01)$ e menor circunferência da panturrilha $(\mathrm{p}$ $<0,01)$.

Conclusão: Este estudo permite concluir que há um alto déficit funcional, diminuição da circunferência da panturrilha, apresentar imobilismo, receber menos visitas e usar bengala/muleta mostraram associação direta com maiores déficits.

Palavras-chave: Idoso. Desempenho físico funcional. Instituição de longa permanência para idosos.

\begin{abstract}
Introduction: Aging progresses with functional changes that predispose the elderly to functional loss, which associated with the institutionalization process is enhanced.

Objective: To assess the level of functional disability of elderly people living in long-term care facilities in Maceió (AL).

Methods: Cross-sectional study carried out with elderly residents in the nine institutions in the city of Maceió. All socioeconomic and demographic data were collected and functional capacity was assessed using the Katz index.

Results: The final sample was 270 individuals. The average age was 78.9 years, with a predominance of females and a low level of education. The statistical analysis showed a significant association between the use of a cane / crutch ( $\mathrm{p}<0.01)$, immobilization syndrome ( $\mathrm{p}<0.01)$, a lower frequency of visits $(\mathrm{p}<0.01)$ and a lower calf circumference $(\mathrm{p}<0.01)$.

Conclusion: This study allows us to conclude that there is a high functional deficit, and a decrease in the calf circumference, presenting immobility, receiving fewer visits and using a cane/crutch showed a direct association with greater deficits.
\end{abstract}

Keywords: Aged. Physical Functional Performance. Homes for the Aged.

\section{Cite como}

Vancouver

Rebêlo, FL, Peixoto, CVG, Lima, JS, Silva, CMA, Santos, AIB. Avaliação e fatores associados à incapacidade funcional de idosos residentes em Instituições de longa permanência. Conscientiae Saúde 2021;20(1):1-11, e18967. https://doi.org/10.5585/conssaude.v20n1.18967. 


\section{Introdução}

Nas últimas décadas, o Brasil e o mundo vivenciam importantes mudanças nos padrões demográficos e de saúde que, entre várias consequências, conduz ao progressivo aumento da população idosa ${ }^{1}$. É importante destacar que o processo de envelhecimento necessita do olhar ampliado em saúde, considerando os aspectos que causam interferência na qualidade de vida dos idosos, como as condições socioeconômicas, o desenvolvimento de comorbidades e a estrutura familiar desses indivíduos ${ }^{2}$.

Desse modo, o cuidado com o indivíduo que envelhece é prioritariamente dos membros da família, tendo, contudo, uma corresponsabilidade do Estado, que deve traçar estratégias que promovam condições adequadas de suporte ao individuo ${ }^{2,3}$, incentivando e promovendo ações, que proporcionam a autonomia e a independência, assim como preconizado pela Política Nacional de Saúde da Pessoa Idosa para um envelhecimento ativo ${ }^{4}$.

No entanto, ressalta-se a necessidade do direcionamento da atenção, também, para aqueles idosos que vivenciam essa fase de vida com dependência e necessidade de cuidados especiais, principalmente aqueles em que a família não possui o suporte adequado para prestar a assistência necessária ${ }^{5}$. Nesse contexto, dentro das modalidades de atenção ao idoso, destacase a importância dos residenciais de cuidado de longa permanência, ambientes de moradia coletiva, de origem pública ou privada, para pessoas com idade igual ou superior a sessenta anos, com ou sem suporte familiar. Nestes locais os indivíduos possuem condições de moradia, assistência profissional e convívio com outros idosos ${ }^{6}$.

No geral, o idoso que é institucionalizado apresenta algum grau de comprometimento funcional, podendo inclusive intensificar-se nos próximos anos, após o processo de institucionalização ${ }^{7}$. Assim, observa-se nessas instituições um maior número de idosos considerados frágeis, que possuem déficits funcionais para o autocuidado e necessidade de auxílio de terceiros para o desempenho de suas atividades de vida diária, gerando a necessidade de uma equipe multidisciplinar qualificada para o cuidado ao idoso dependente, que também atue preventivamente para evitar a progressão acelerada da incapacidade funcional ${ }^{6}$.

Contudo, a realidade para a maioria das ILPI's no Brasil difere bastante do que é preconizado pela diretoria colegiada da Agência Nacional de Vigilância Sanitária (ANVISA). Aspetos como o isolamento social, por exemplo, comum em boa parte das instituições, acabam interferindo negativamente no status funcional do idoso, conduzindo a importantes limitações em virtude de comprometimentos da função motora e cognitiva, tornando o idoso cada vez mais dependente ${ }^{8}$. 
Dessa forma, compreendendo os componentes que levam a um envelhecimento bemsucedido, como independência nas atividades de vida diária, relações psicossociais e condições socioeconômicas, torna-se relevante e necessário a investigação dos fatores associados à capacidade funcional do idoso residente em instituições de longa permanência ${ }^{8}$, sendo possível, com isso, um melhor direcionamento de ações voltadas a essa população. Assim, o presente estudo teve por objetivo avaliar o nível e os fatores associados à incapacidade funcional de idosos institucionalizados

\section{Métodos}

Trata-se de um estudo observacional, de corte transversal, que teve protocolo de pesquisa aprovado pelo de Comitê de Ética em Pesquisa do Centro Universitário Cesmac, sob o número do parecer: 051406/2015. O estudo foi realizado em todas as ILP's de Maceió (Alagoas), dentro das dependências físicas das instituições. Sendo a amostra final de 270 idosos.

Para participação da pesquisa, o consentimento foi mediante assinatura do Termo de Consentimento Livre e Esclarecido e para aqueles que não respondiam sobre si, a autorização foi concedida pelo responsável legal. Foram incluídos todos os idosos com idade igual ou superior a sessenta anos, de ambos os sexos, residentes em instituições de longa permanência do município de Maceió, Alagoas, sendo excluídos aqueles que, por ventura, utilizassem a instituição apenas na modalidade de Centro Dia.

A coleta foi iniciada pelos dados socioeconômicos, demográficos e referentes à saúde nas fichas cadastrais e/ou prontuários. Em seguida, foram realizadas as avaliações específicas e aplicações dos questionários com os idosos. Para os dados que não constavam nas fichas, o preenchimento, quando possível, era realizado diretamente com o idoso, cuidador formal, familiar ou com os funcionários das ILPI's.

A avaliação socioeconômica e demográfica constou de dados como: sexo, faixa etária, escolaridade, estado civil, renda e de saúde, quanto ao número de morbidades, de medicamentos, prática de atividade física e repercussões funcionais, e uma avaliação funcional realizada através do índice de Katz.

O Índice de independência nas atividades de vida diária (Índice de Katz), foi desenvolvido por Sidney Katz e publicado pela primeira vez em 1963. É composto pela avaliação das atividades de alimentação, vestir-se, continência, transferência, ir ao banheiro e banhar-se, classificando o idoso em níveis de dependência de acordo com o número de atividades comprometidas, numa escala que varia de zero (independente para as seis atividades 
básicas de vida diária) a seis (dependente para as seis atividades básicas de vida diária) ${ }^{9}$. A avaliação era realizada através da observação direta, pelos pesquisadores, e complementada e/ou confirmada com os próprios idosos e/ou cuidadores, quando necessário.

As variáveis contínuas estão apresentadas como média, desvio-padrão, e valores mínimos e máximos. Já as variáveis categóricas estão apresentadas como frequências relativas e absolutas. A variável KATZ apresenta-se de maneira adequada como uma contagem, variando de zero a seis, por isso, foi analisada por meio da regressão de Poisson, onde para as razões de taxas foram calculados os intervalos de confiança de 95\%. Para todas as análises de regressão, primeiro foram conduzidas diversas regressões univariadas, incluindo as variáveis preditoras uma por vez e aquelas variáveis que apresentaram $\mathrm{p}<0,20$ foram incluídas num modelo multivariado, para verificar quais mantinham significância estatística. Em todas as análises foi utilizado um valor de alfa igual a 5\% e o auxílio do programa estatístico SPSS v 20.0.

\section{Resultados}

Foram identificados 309 idosos residentes nas nove ILP's existentes em Maceió, no período de realização da coleta de dados, que aconteceu entre os anos de 2015 e 2016. Trinta idosos não consentiram a participação no estudo e 9 encontravam-se hospitalizados durante a coleta. Dessa forma, a amostra final foi de 270 idosos. Para esse estudo analisaram-se apenas os dados referentes à avaliação da capacidade funcional através do índice de Katz.

A idade média dos indivíduos da pesquisa foi de 78,9 anos $( \pm 9,8)$, com mínima de sessenta e máxima de 113 anos. Houve predomínio do sexo feminino 158 (41,5\%) e identificouse um baixo nível de escolaridade, com média de 2,6 anos de estudo $( \pm 3,7)$. Todos os dados referentes às características socioeconômicas e demográficas estão descritas na Tabela 1. 
Tabela 1 - Distribuição da frequência das variáveis sócio demográficas em idosos institucionalizados na cidade de Maceió

\begin{tabular}{lll}
\hline Variável & Frequência & $\%$ \\
\hline Sexo & 112 & 41,48 \\
M & 158 & 58,52 \\
F & & \\
Faixa etária & 46 & 17,03 \\
$60-69$ & 93 & 34,44 \\
$70-79$ & 91 & 33,70 \\
$80-89$ & 32 & 11,86 \\
$90-99$ & 5 & 1,86 \\
$>100$ & 3 & 1,11 \\
NC* & & \\
Escolaridade & 118 & 43,70 \\
Analfabeto & 54 & 20,00 \\
$<4$ anos & 29 & 10,75 \\
$5-8$ Anos & 20 & 7,40 \\
$>9$ anos & 49 & 18,15 \\
NC* & & \\
Estado Civil & 106 & 39,26 \\
Solteiro & 40 & 14,81 \\
Divorciado & 15 & 5,56 \\
Casado & 67 & 24,81 \\
Viúvo & 42 & 15,56 \\
NC* & 114 & 42,22 \\
NC* & & \\
NC & &
\end{tabular}

*NC $=$ não consta

Fonte: Autor.

Quanto à avaliação funcional realizada através do Índice de Katz, observou-se um alto nível de dependência entre os idosos avaliados, como demonstrado na Tabela 2.

Tabela 2 - Nível de dependência funcional dos idosos institucionalizados segundo índice de Katz, em Maceió-AL

Níveis de dependência no Índice de KATZ \%
Dependentes para 5 ou 6 AVD'S
51,1
Dependentes para 3 a 4 AVD'S
10,3
Dependentes para 1 ou 2 AVD'S
19,4
Independente
19,3

Fonte: Autor.

A correlação da variável de capacidade funcional avaliada pelo Katz e as características socioeconômicas e demográficas estão descritas na Tabela 3, onde verificou-se que quatro 
variáveis apresentaram associação com o escore de KATZ. Dentre os aspectos que geraram aumento na contagem, ressalta-se que os indivíduos que usavam bengala/muleta apresentaram aumento de $43 \%$ e os indivíduos com imobilismo apresentaram mais de $200 \%$ de aumento na contagem. Quanto aos aspectos que produziram influência na redução da contagem do índice, observa-se que os idosos que recebiam visitas apresentaram uma redução de $33 \%$ e para cada aumento de $1 \mathrm{~cm}$ na panturrilha, encontrou-se uma redução de 5\% na contagem de KATZ.

Tabela 3 - Fatores associados à incapacidade funcional em idosos institucionalizados na cidade de Maceió

\begin{tabular}{|c|c|c|c|c|c|c|}
\hline \multirow[t]{2}{*}{ Variável } & \multicolumn{3}{|c|}{ Univariada } & \multicolumn{3}{|c|}{ Multivariada $^{1}$} \\
\hline & RT & IC $95 \%$ & P-valor & RT & IC 95\% & $\begin{array}{c}\text { P- } \\
\text { valor }\end{array}$ \\
\hline Sexo Masculino & 0,71 & $0,59-0,87$ & $<0,01$ & 0,78 & $0,60-1,01$ & 0,06 \\
\hline Idade (anos) & 1,01 & $1,00-1,02$ & $<0,01$ & 0,99 & $0,98-1,01$ & 0,52 \\
\hline Casado & 0,91 & $0,58-1,44$ & 0,70 & - & - & - \\
\hline Anos de estudo & 0,98 & $0,94-1,02$ & 0,41 & - & - & - \\
\hline Renda > 1 salário & 1,04 & $0,65-1,68$ & 0,84 & - & - & - \\
\hline Tem doença & 1,07 & $0,85-1,34$ & 0,54 & - & - & - \\
\hline Tabagista & 0,62 & $0,42-0,91$ & 0,01 & 0,67 & $0,40-1,11$ & 0,12 \\
\hline Etilista & 1,10 & $0,73-1,67$ & 0,63 & - & - & - \\
\hline Hobbies & 0,70 & $0,54-0,90$ & $<0,01$ & 0,86 & $0,64-1,15$ & 0,32 \\
\hline Número de morbidades & 0,94 & $0,82-1,08$ & 0,40 & - & - & - \\
\hline Saúde atual boa/excelente & 0,70 & $0,35-1,39$ & 0,32 & - & - & - \\
\hline Saúde melhor que há 1 ano & 0,95 & $0,69-1,30$ & 0,75 & - & - & - \\
\hline Amputação & 1,43 & $1,01-2,01$ & 0,03 & 1,47 & $0,96-2,27$ & 0,07 \\
\hline Uso de bengala/muleta & 1,21 & $0,99-1,49$ & 0,05 & 1,43 & $1,11-1,85$ & $<0,01$ \\
\hline Pratica atividade física & 0,12 & $0,03-0,52$ & $<0,01$ & 0,25 & $0,06-1,07$ & 0,06 \\
\hline Recebe visitas & 0,74 & $0,58-0,94$ & 0,01 & 0,67 & $0,51-0,89$ & $<0,01$ \\
\hline Tem fraturas & 0,98 & $0,76-1,28$ & 0,92 & - & - & - \\
\hline Número de medicações & 0,98 & $0,94-1,01$ & 0,27 & - & - & - \\
\hline Panturrilha $(\mathrm{cm})$ & 0,94 & $0,92-0,95$ & $<0,01$ & 0,95 & $0,93-0,98$ & $<0,01$ \\
\hline Usa prótese auditiva & 0,73 & $0,29-1,84$ & 0,51 & - & - & - \\
\hline
\end{tabular}




$\begin{array}{lcccccc}\text { Conversação adequada } & 1,37 & 1,10-1,71 & <0,01 & 1,09 & 0,85-1,41 & 0,46 \\ \text { Usa lentes corretivas } & 0,70 & 0,54-0,92 & 0,01 & 0,85 & 0,65-1,11 & 0,24 \\ \text { Leitura adequada } & 1,06 & 0,82-1,38 & 0,61 & - & - & - \\ \text { Imobilismo } & 1,88 & 1,69-2,08 & <0,01 & 2,30 & 1,83-2,89 & <0,01 \\ \text { Já caiu } & 0,80 & 0,63-1,02 & 0,07 & 1,03 & 0,80-1,32 & 0,80 \\ \text { Repercussão funcional } & 0,95 & 0,67-1,35 & 0,80 & - & - & - \\ \text { Repercussão funcional } & 0,95 & 0,67-1,35 & 0,80 & - & - & -\end{array}$

RT, razão de taxas; IC, Intervalo de confiança

${ }^{1}$ Modelo de regressão de Poisson multivariada que incluiu todas as variáveis que apresentaram $\mathrm{P}<0,20$ na análise univariada.

Fonte: Autor.

\section{Discussão}

O estudo aqui apresentado identificou questões relevantes de enfoque gerontológico para a população de idosos residentes em ILPI's numa capital nordestina. A alta prevalência de incapacidade funcional e a associação com fatores de ordem física, como massa muscular e de ordem social, como visitação, chamam a atenção a respeito da multidimensionalidade relacionada a essa variável tão importante para a manutenção da qualidade de vida dos idosos.

A caracterização do perfil socioeconômico e demográfico revelou uma população predominantemente feminina de idade avançada, com baixa escolaridade e renda, refletindo a realidade da maioria dos idosos nordestinos sendo em sua maioria solteiras, dados estes corroborados por vários estudos que também avaliaram populações semelhantes ${ }^{10,11,12}$.

Ao analisar a capacidade funcional, que, em termos gerais, é caracterizada pela habilidade de realizar as atividades de vida diária com independência e autonomia, entende-se que durante o processo de envelhecimento, principalmente quando mal sucedido, essa variável sofre paulatina diminuição ${ }^{13,14}$. Sendo evidenciado pelos achados no presente estudo ao observar o alto nível de incapacidade funcional presente nesta população. Diante disso, ao serem observados os aspectos que podem interferir na redução da capacidade funcional, percebeu-se que esse desfecho pode ser influenciado por diferentes tipos de fatores, inclusive, sociais.

$\mathrm{O}$ risco de comprometimento funcional para idosos que vivem sem parceiros e socialmente mais isolados. O cuidado advindo do parceiro no âmbito da saúde, alimentação e atividades de lazer contribuem para a melhora ou manutenção da capacidade funcional. Quanto ao isolamento social, indivíduos com sintomas depressivos tendem a apresentar cansaços e mal- 
estar, que levam à dificuldade na socialização, impactando nos idosos em sua capacidade funcional ${ }^{15}$.

Ou seja, o viver sozinho, sem contato frequente com amigos ou familiares, invariavelmente reduz a sociabilidade dos idosos, tornando-os predispostos a sintomas depressivos, por exemplo, condições que são significativamente potencializadas no contexto da institucionalização, visto que muitos não são visitados pelos familiares por longos períodos isolando-se cada vez mais ${ }^{16}$. Desse modo, corroborando com os resultados encontrados neste estudo, onde observou-se que houve uma redução de 33\% no índice de Katz para os indivíduos que recebiam visitas de seus familiares.

Além disso, no processo de envelhecimento quando há um importante comprometimento funcional a uma tendência progressiva ao uso de dispositivos de auxílio funcional, como demonstrado neste estudo, em que houve um maior declínio funcional em idosos que utilizavam muletas ou bengalas, com o aumento no índice de $43 \%$ para os indivíduos que utilizavam estes dispositivos. Posto isto, a redução das aptidões como força, agilidade, flexibilidade e coordenação interferem na funcionalidade do idoso, gerando dependência de dispositivos de auxílio para marcha ${ }^{17}$.

Ademais, estas reduções de aptidões relacionam-se com a diminuição da velocidade da marcha, fisiologicamente presente no envelhecimento, e com o medo de cair, sendo estes dois fatores também importantes influenciadores no declínio funcional. Nas residências de cuidado de longa permanência isto é intensificado, por nestes ambientes haver uma redução da socialização, da independência principalmente desses idosos que apresentam diminuição da velocidade da marcha e medo de cair e consequentemente uma redução de mobilidade ${ }^{18,19}$.

No entanto, ressalta-se que o envelhecimento não se caracteriza como um processo patológico. Contudo, nessa fase existe um maior número de fatores que interferem no status funcional do indivíduo, como a presença de comorbidades, alterações musculares e no convívio social que levam a uma maior dependência funcional. Podendo esse aumento levar ao desenvolvimento da Síndrome do Imobilismo (SI), condicionando o idoso à necessidade de auxílio humano e de equipamentos para a realização das atividades ${ }^{20}$

Considerando a SI, no estudo aqui apresentado, houve aumento de $200 \%$ no índice de Katz para os idosos que a possuíam, o que é extremamente esperado numa associação com um desfecho intimamente relacionado com a mobilidade, como mencionado no estudo de Silva, Filoni e Suguimoto $^{21}$, que verificaram que o imobilismo impacta em prejuízos no desenvolvimento e execução das atividades de vida diárias (AVDs) de forma independente. Por fim, ao avaliar a circunferência da panturrilha, foi identificada associação entre diminuição da 
massa muscular e uma maior dependência funcional, corroborando com Spinelli ${ }^{22}$, que avaliou idosos independentes institucionalizados em Erechim (RS), identificando que a circunferência é menor nos idosos residentes em ILP's.

A capacidade funcional no idoso pode ser influenciada direta ou indiretamente por diversos fatores, de ordem física, psíquica e social. Quando o envelhecimento é vivenciado com doenças associadas, em nível de comprometimento funcional será ainda maior, quadros esses característicos entre os idosos institucionalizados. É preciso, portanto, compreender, avaliar e intervir sobre todos os aspectos que permeiam o desfecho da capacidade funcional, entendendo que essa é condição sine qua non para a manutenção/otimização da qualidade de vida do idoso, desfecho final de qualquer intervenção de ordem gerontológica.

Ademais, salienta-se a relevância do papel da família na institucionalização, uma vez que a presença da família na vida desses indivíduos, contribui para a manutenção da funcionalidade e, consequentemente, qualidade de vida. Por fim, destaca-se as limitações deste estudo, ressaltando-se a importância da realização de pesquisas de corte longitudinal, àqueles que busquem identificar causa e efeito, já que os estudos de corte transversal, apesar de importantes, limitam-se a análises temporais que representam apenas o momento avaliado.

\section{Conclusão}

Os achados encontrados neste estudo permitiram concluir um alto nível de incapacidade funcional para a amostra estudada, visto que 51,1\% configuraram-se como dependentes para 5 ou 6 AVD's, de acordo com o Índice de Katz. Além disso, essa variável apresentou associação com uso de muleta ou bengala, imobilismo, diminuição da massa muscular na panturrilha e menor frequência de visitas. 


\section{Referências}

1. IBGE - Instituto Brasileiro de Geografia e Estatística. Censo demográfico: Características da população e dos domicílios. Rio de Janeiro; 2011.

https://biblioteca.ibge.gov.br/visualizacao/periodicos/93/cd_2010_caracteristicas_populacao_ domicilios.pdf

2. Miranda GMD, Mendes ACG, Silva ALA. O envelhecimento populacional brasileiro: desafios e consequências sociais atuais e futuras. Rev. bras. geriatr. gerontol. 2016;19(3):507519. http://dx.doi.org/10.1590/1809-98232016019.150140.

3. Camarano AA, Kanso S. As instituições de longa permanência para idosos no Brasil. R Bras Est Pop. 2010;27(1):233-235. https://doi.org/10.1590/S0102-30982010000100014.

4. Romero DE, Pires DC, Marques A, Muzy J. Diretrizes e indicadores de acompanhamento das políticas de proteção à saúde da pessoa idosa no Brasil. Rev Eletron Comun Inf Inov Saúde. 2019;13(1):157-135. https://doi.org/10.29397/reciis.v13i1.1569.

5. Costa MCNS, Mercadante EF. O Idoso residente em ILPI (Instituição de Longa Permanência do Idoso) e o que isso representa para o sujeito idoso. Revista Kairós Gerontologia. 2013;16(2):209-222. https://doi.org/10.23925/2176-901X.2013v16i1p209-222

6. Costa SDM, Soares LU, Fernandes AK, Braz EC, Costa SH, Freire APAC. Fragilidade e status funcional de idosos institucionalizados. Revista de Pesquisa Cuidado é Fundamental Online. 2015;7(3):2688-2696. https://doi.org/10.9789/2175-5361.2015.v7i3.2688-2696.

7. Tonatto FR. Idosos em instituições de longa permanência: Uma revisão de estudos nacionais. Caxias do Sul: Universidade de Caxias do Sul, 2019. Trabalho de Conclusão de Curso em Psicologia. https://repositorio.ucs.br/11338/5023

8. Lisboa CR, Chianca TCM. Perfil epidemiológico, clínico e de independência funcional de uma população idosa institucionalizada. Rev. bras. enferm. 2012;65(3):482-488. https://doi.org/10.1590/S0034-71672012000300013.

9. Soares RR, Salgado PO, Rocha KO, Lima LM. Functional capacity and risk of falls in the elderly with metabolic syndrome. Rev Bras Med Fam Comunidade. 2020; 15(42):2228. https://doi.org/10.5712/rbmfc15(42)2228

10. Duarte MCS, Lima US, Albuquerque KF, Evangelista CB, Souto HC, Patrício ACFA. Fragilidade e status funcional de idosos institucionalizados. J. res.: fundam. care. 2015;7(3):2688-2696. http://dx.doi.org/10.9789/2175-5361.2015.v7i3.2688-2696.

11. Camarano AA, Barbosa P. Instituições de Longa Permanência para Idosos no Brasil: do que se está falando? In: Alcântara AO, Camarano A A, Giacomin KC, orgs. Política Nacional do Idoso: velhas e novas questões. Rio de Janeiro: Ipea; 2016. p. 479-514.

http://repositorio.ipea.gov.br/bitstream/11058/9146/1/Institui\%C3\%A7\%C3\%B5es\%20de\%2 0longa\%20perman\%C3\%AAncia.pdf 
12. Alcântara R, Cavalcante M, Fernandes B, Lopes V, Leite S, Borges C. Perfil Sociodemográfico de Saúde de Idosos Institucionalizados. Journal of Nursing, 2019;13(3): 674-679. https://doi.org/10.5205/1981-8963-v13i03a237384p674-423-2019.

13. Botoluzzi EC, Doring M, Portella M, Cavalcanti G, Mascarelo A, Dellani M. Prevalência e fatores associados à dependência funcional em idosos longevos. Rev Bras Ativ Fís Saúde. 2017;22(1):85-94. https://doi.org/10.12820/rbafs.v.22n1p85-94

14. Berlezi EM, Farias AM, Dallazen F, Oliveira KR, Pillatt AP, Fortes CK. Analysis of the functional capacity of elderly residents of communities with a rapid population aging rate. Rev. bras. geriatr. gerontol. 2016;19(4):643-652. https://doi.org/10.1590/180998232016019.150156

15. Matos FS, Jesus CS, Carneiro JAO, Coqueiro RS, Fernandes MH, Brito TA. Redução da capacidade funcional de idosos residentes em comunidade: estudo longitudinal. Ciência \& Saúde Coletiva. 2018;23(10):3393-3401. https://doi.org/10.1590/1413812320182310.23382016

16. Silva JKSD, Albuquerque MCDS, Souza EMSD, Monteiro FS, Esteves GGL. Sintomas depressivos e capacidade funcional em idosos institucionalizados. Cult Cuid. 2015;41:157-7. https://rua.ua.es/dspace/bitstream/10045/46623/1/Cultura-Cuidados_41_18.pdf.

17. Benavides-Rodríguez CL, García-García JA, Fernández JA. Condición física funcional en adultos mayores institucionalizados. Univ. Salud. 2020;22(3):238-245. https://doi.org/10.22267/rus.202203.196

18. Utida KAM, Budib MB, Batiston AP. Medo de cair associado a variáveis sociodemográficas, hábitos de vida e condições clínicas em idosos atendidos pela Estratégia de Saúde da Família em Campo Grande-MS. Rev. bras. geriatr. gerontol. 2016;19(3):441452. https://doi.org/10.1590/1809-98232016019.150069.

19. Lenardt MH, Setoguchi LS, Betiolli SE, Grden CRB, Sousa JAV, Lourenço TM. Gait speed and incidence of falls in the long-lived elderly. REME - Rev Min Enferm. 2019;23:e1190. https://doi.org/10.5935/1415-2762.20190038

20. Certo AC, Sanchez K, Galvão A, Fernandes H. A síndrome da fragilidade nos idosos: revisão da literatura. Actas Gerontol. 2016;(1):1-11. http://hdl.handle.net/10198/12983

21. Silva JL, Filoni E, Suguimoto CM. Análise do incremento da força muscular para reaquisição de ortostatismo em idosos com síndrome do imobilismo temporário. Acta fisiátrica. 2017;24(3):113-119. https://doi.org/10.5935/0104-7795.20170021

22. Sperotto FM, Spinelli, RB. Avaliação nutricional em idosos independentes de uma Instituição de Longa permanência no município de Erechim-rs. PERSPECTIVA. 2010;34(125):105-116. http://www.uricer.edu.br/new/site/pdfs/perspectiva/125_78.pdf 